\title{
A Deep Radio Limit for the TRAPPIST-1 System
}

\author{
J. Sebastian Pineda ${ }^{1}$ (1) and Gregg Hallinan ${ }^{2}$ \\ ${ }^{1}$ University of Colorado Boulder, Laboratory for Atmospheric and Space Physics, 3665 Discovery Drive, \\ Boulder, CO 80303, USA; sebastian.pineda@lasp.colorado.edu \\ ${ }^{2}$ California Institute of Technology, Department of Astronomy, 1200 E. California Avenue, Pasadena, CA 91125, USA \\ Received 2018 June 1; revised 2018 September 6; accepted 2018 September 6; published 2018 October 24
}

\begin{abstract}
The first nearby very-low-mass star-planet-host discovered, TRAPPIST-1, presents not only a unique opportunity for studying a system of multiple terrestrial planets, but a means to probe magnetospheric interactions between a star at the end of the main sequence and its close-in satellites. This encompasses both the possibility of persistent coronal solar-like activity, despite cool atmospheric temperatures, and the presence of large-scale magnetospheric currents, similar to what is seen in the Jovian system. Significantly, the current systems include a crucial role for close-in planetary satellites analogous to the role played by the Galilean satellites around Jupiter. We present the first radio observations of the seven-planet TRAPPIST-1 system using the Karl G. Jansky Very Large Array, looking for both highly circularly polarized radio emission and/or persistent quiescent emissions. We measure a broadband upper flux density limit of $<8.1 \mu \mathrm{Jy}$ across $4-8 \mathrm{GHz}$, and place these observations both in the context of expectations for stellar radio emission, and the possible electrodynamic engines driving strong radio emissions in very-low-mass stars and brown dwarfs, with implications for future radio surveys of TRAPPIST-1 like planethosts. We conclude that the magnetic activity of TRAPPIST-1 is predominantly coronal and does not behave like the strong radio emitters at the stellar/substellar boundary. We further discuss the potential importance of magnetic field topology and rotation rates, demonstrating that a TRAPPIST-1 like planetary system around a rapidly rotating very-low-mass star can generate emission consistent with the observed radio luminosities of very-low-mass stars and brown dwarfs.
\end{abstract}

Key words: brown dwarfs - planets and satellites: aurorae - stars: activity - stars: individual (TRAPPIST-1)

\section{Introduction}

Recently, Gillon et al. (2017) announced the detection of a seven-planet system, including three Earth-sized planets in the liquid water habitable zone, orbiting a nearby very-low-mass star, TRAPPIST-1. The system is particularly exciting because it provides appealing targets for ongoing and future transmission spectroscopy observations, and a way to conduct comparative exoplanetology in terrestrial bodies around a single host star (e.g., Barstow \& Irwin 2016; Morley et al. 2017; de Wit et al. 2018). This system also probes planet formation around the lowest mass stars (e.g., Alibert \& Benz 2017), and will be a benchmark for characterizing exoplanets with properties similar to Earth (Gillon et al. 2017).

Interestingly, TRAPPIST-1 resides in the regime of ultracool dwarfs (UCDs; spectral type $\gtrsim \mathrm{M} 7$ ) at the end of the main sequence. These objects exhibit a transition with cooler effective temperature from coronal stellar activity, as is seen on more massive stars, to magnetic emissions driven by largescale magnetospheric currents like those that power the multiwavelength aurorae of Jupiter as well as some brown dwarfs (Pineda et al. 2017). While many low-mass stars and late-type $\mathrm{M}$ dwarfs, exhibit the X-ray emission, stellar winds, UV transition region spectral lines, chromospheric $\mathrm{H} \alpha$, and radio emission properties consistent with the coronal solar-like paradigm (see within Pineda et al. 2017), there is a distinct subpopulation of very-low-mass stars whose magnetic activity indicators show very different properties; specifically, extensive evidence for rotationally pulsed electron cyclotron maser (ECM) emission, consistent with the presence of magnetospheric auroral currents (see within Pineda et al. 2017). In the late $\mathrm{L}$ dwarf and $\mathrm{T}$ dwarf regime, these auroral processes dominate the magnetic activity (Pineda et al. 2016, 2017). This population is most clearly evident through observations revealing much stronger (several orders of magnitude) radio emission from these objects than would be expected based on the empirical Güdel-Benz relation connecting coronal X-ray and radio emissions in low-mass stars (Guedel \& Benz 1993; Williams et al. 2014), as well as the presence of periodic highly circularly polarized emission. Although it is unclear what the underlying conditions are that drive the presence of large-scale magnetospheric currents in these objects, it is likely related to rapid rotation rates, and strong large-scale magnetic fields (Pineda et al. 2017; Turnpenney et al. 2017). The presence of auroral radio emission might then be related to the bistability of magnetic dynamos in fully convective low-mass stars as seen through Zeeman Doppler Imaging (ZDI), which show either strong dipolar large-scale fields or weaker nonaxisymmetric multipolar fields (Morin et al. 2010), with the strongest average field strengths observed among the fastest rotating objects with predominantly dipolar field topologies (Shulyak et al. 2017). If auroral radio processes require these large-scale dipolar fields, then the aurorae could be indicative of which branch of the dynamo regime a given object may inhabit. Moreover, the prevalence of a given fully convective dynamo branch would then influence the statistics of auroral radio detections. Understanding this connection could provide a new means to probe fully convective dynamos in low-mass stars.

Fast rotation and high field strengths may be necessary conditions to drive significant auroral magnetospheric currents; however, the nature of the underlying electrodynamic engine powering the current system in these UCDs remains an open question. Possible hypotheses include magnetospheric-ionospheric coupling currents driven by the large-scale motions of an equatorial plasma disk (Schrijver 2009; Nichols et al. 2012; 
Turnpenney et al. 2017), reconnection between the large-scale magnetosphere and the interstellar medium (Turnpenney et al. 2017), or the electrodynamic interaction between the UCD's magnetic field and any nearby orbiting satellites (Hallinan et al. 2015; Pineda et al. 2017). Models of these processes suggested that they can generate sufficient energy to power the observed emissions (Zarka 2007; Schrijver 2009; Nichols et al. 2012; Saur et al. 2013; Turnpenney et al. 2017), but it remains unclear which of these mechanisms, or what mixture of them, are predominately responsible for generating the auroral ECM emission of some UCDs. Similarly, the origins of the quiescent radio emission in this same population of radio UCDs is uncertain. This emission is likely synchrotron or gyrosynchrotron radiation and may be associated with high energy electrons trapped in closed magnetospheric loops, akin to the Jovian radiation belts (Hallinan et al. 2006; Pineda et al. 2017). However, the continual mass-loading of the magnetosphere with plasma, a requirement for both the existence of the plasma radiation belts and several of the proposed electrodynamics engines is itself an open question, and might be related to volcanic planetary activity, similar to the Jovian system, or possibly atmospheric sputtering (Hallinan et al. 2015).

As the only known nearby UCD planet-host, TRAPPIST-1 provides the first opportunity to test whether the presence of a close-in exoplanetary system plays a significant role in generating these magnetic emission processes around verylow-mass stars and brown dwarfs, possibly through a direct interaction with the stellar host or by providing the magnetospheric plasma source. By observing TRAPPIST-1 at radio wavelengths, we can look for the possibility of ECM emission or quiescent radio emission, and assess whether its activity properties (e.g., radio, X-ray, and $\mathrm{H} \alpha$ ) are consistent with the coronal solar-like paradigm or whether it may belong to the subpopulation of very-low-mass stars exhibiting auroral phenomena. Understanding how this stellar system, with potentially significant interactions with its planetary satellites, fits into the transition in magnetic activity indicators in the UCD regime thus motivates the current radio study of this verylow-mass star. This article is organized as follows. In Section 2, we review the properties of TRAPPIST-1. In Section 3, we discuss our data set from the NSF's Karl G. Jansky Very Large Array (VLA; Perley et al. 2011). In Section 4, we compare our observations to estimates of the stellar radio flux densities from TRAPPIST-1. In Section 5, we discuss the role of satellites for possible auroral emission mechanisms in UCDs. Lastly, in Section 6, we summarize our findings with implications for future searches of radio emission at the end of the main sequence.

\section{TRAPPIST-1}

TRAPPIST-1, also know as 2MASS J23062928-0502285, is an M8 dwarf at $12.1 \mathrm{pc}$ which hosts seven terrestrial planets, detected in transit from photometric monitoring (Gillon et al. 2016, 2017; Luger et al. 2017). The star has observed variable $\mathrm{H} \alpha$ and $\mathrm{Ly} \alpha$ emission with typical levels of $\log _{10}\left(L_{\mathrm{H} \alpha} / L_{\mathrm{bol}}\right) \sim-4.7$ (Burgasser \& Mamajek 2017) and $\log _{10}\left(L_{\mathrm{Ly} \alpha} / L_{\text {bol }}\right) \sim-4.1$ (Bourrier et al. 2017), respectively, and displays photometric variability at optical wavelengths from $K 2$ monitoring with a periodicity of 3.295 days (Luger et al. 2017; Vida et al. 2017). This updated rotation period differs from the initially published period in Gillon et al. (2016) of 1.4 days, but is consistent with the updated projected
Table 1

Properties of the UCD TRAPPIST-1

\begin{tabular}{|c|c|c|}
\hline Property & Value & References \\
\hline Spectral Type ... & M8 & (1) \\
\hline Distance ... & $12.14 \pm 0.12 \mathrm{pc}$ & (2) \\
\hline Mass ... & $0.089 \pm 0.006 M_{\odot}$ & (2) \\
\hline Radius ... & $0.121 \pm 0.003 R_{\odot}$ & (2) \\
\hline$L_{\mathrm{bol}} \ldots$ & $5.22 \pm 0.19 \times 10^{-4} L_{\odot}$ & (2) \\
\hline$T_{\text {eff }} \cdots$ & $2516 \pm 41 \mathrm{~K}$ & (2) \\
\hline Rotation Period $^{\mathrm{a}} \ldots$ & $3.295 \pm 0.003$ days & (3) \\
\hline$v \sin i \ldots$ & $<2 \mathrm{~km} \mathrm{~s}^{-1}$ & (4) \\
\hline$B f^{\mathrm{b}} \ldots$ & $600 \pm_{400}^{200} \mathrm{G}$ & (5) \\
\hline$L_{\mathrm{X}} \ldots$ & $3.8-7.9 \times 10^{26} \mathrm{erg} \mathrm{s}^{-1}$ & (6) \\
\hline $\log _{10}\left(L_{\mathrm{Ly} \alpha} / L_{\mathrm{bol}}\right) \ldots$ & $\sim-4.1$ & (7) \\
\hline $\log _{10}\left(L_{\mathrm{H} \alpha} / L_{\mathrm{bol}}\right) \ldots$ & $\sim-4.7$ & (8) \\
\hline$L_{\nu}(4-8 \mathrm{GHz}) \ldots$ & $<1.43 \times 10^{12} \mathrm{erg} \mathrm{s}^{-1} \mathrm{~Hz}^{-1}$ & (9) \\
\hline
\end{tabular}

Notes.

a Rotation period is taken from $K 2$ photometric variability.

b Denotes the surface averaged magnetic field strength from Zeeman Broadening measurements; $f$ is the filling factor between 0 and 1 .

References. (1) Burgasser et al. (2015); (2) Van Grootel et al. (2018); (3) Vida et al. (2017); (4) Reiners et al. (2018); (5) Reiners \& Basri (2010); (6) Wheatley et al. (2017); (7) Bourrier et al. (2017); (8) Burgasser \& Mamajek (2017); (9) this paper.

rotational velocity of $v \sin i<2 \mathrm{~km} \mathrm{~s}^{-1}$ (Reiners et al. 2018); ${ }^{3}$ Roettenbacher \& Kane (2017) discussed the discrepancy in the period measurements, attributing the different results to changing stellar surface features (also see Morris et al. 2018, who consider the possibility of bright surface spots generating the $\sim 3.3$ day periodogram signal). XMM Newton observations have also detected an X-ray luminosity of $(3.8-7.9) \times 10^{26} \mathrm{erg} \mathrm{s}^{-1}$ in the band $0.1-2.4 \mathrm{keV}$, which is consistent with observations of other late $\mathrm{M}$ dwarfs, although among the strongest such emitters (Williams et al. 2014; Wheatley et al. 2017). The stellar physical properties and emission characteristics of TRAPPIST-1 are summarized in Table 1, using the values based on new parallax measurements from Van Grootel et al. (2018).

\section{Observations}

In order to categorically rule out periodic bright radio pulsations from TRAPPIST-1, we would need to monitor the star for a duration exceeding its rotation period, as well as the orbital period of the inner planets. However, in the case of all known periodically pulsing UCDs, the pulsed emission is accompanied by a quiescent radio component, favoring a short initial search for quiescent emission as a proxy for the ECM induced emissions (Pineda et al. 2017). We thus conducted an initial pilot observation of TRAPPIST-1 with the VLA (project 16A-466, PI Pineda) on 2016 May 12 from UT 12:57:37 to 14:57:16 in the $\mathrm{CnB}$ configuration, to look for circularly polarized pulses and measure a potential quiescent radio component. We chose to observe at C-band $(4-8 \mathrm{GHz})$ due to the success of previous volume-limited radio surveys in detecting UCD radio emission between 4 and $10 \mathrm{GHz}$ (Antonova et al. 2013), as well as evidence that the quiescent radio luminosity of this population broadly peaks in this band

\footnotetext{
3 There was some tension in these measurements initially with $v \sin i=$ $6 \pm 2 \mathrm{~km} \mathrm{~s}^{-1}$ (Reiners \& Basri 2010).
} 

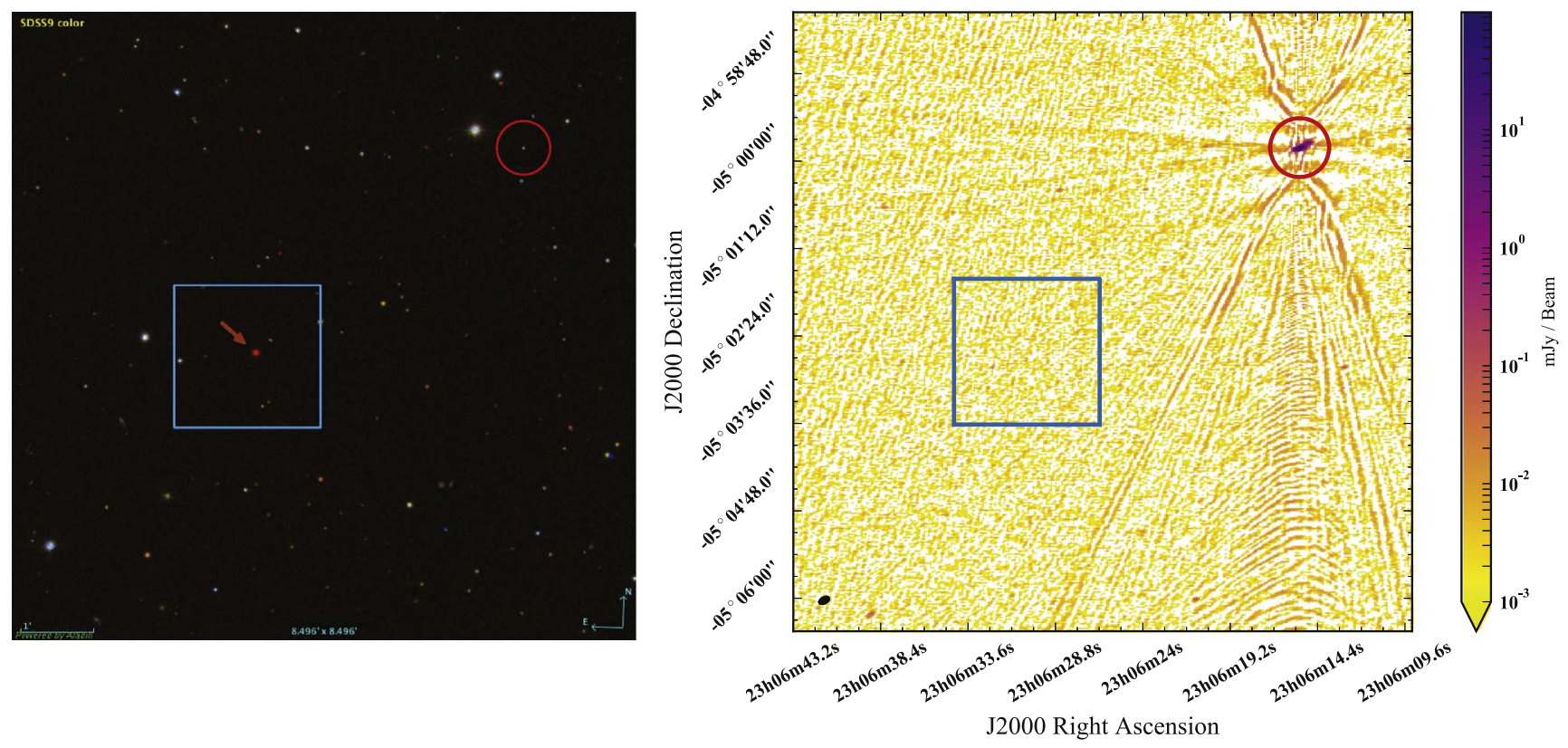

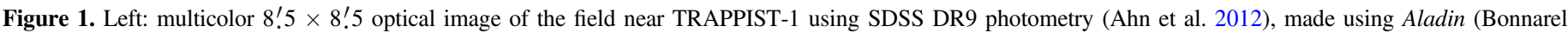

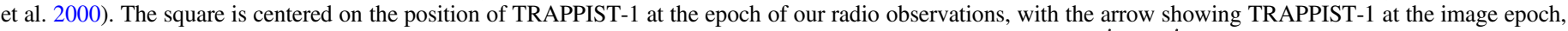

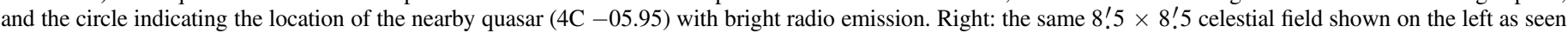

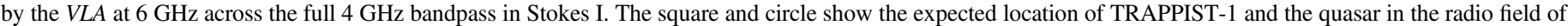

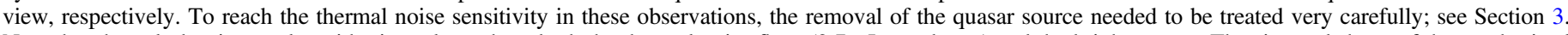

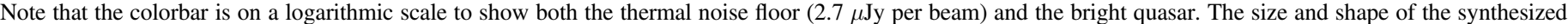
beam is shown in the lower left.

(Ravi et al. 2011; Williams et al. 2015a). Observations were carried out using the $4 \mathrm{GHz}$ bandwidth enabled by the three-bit sampler mode of the WIDAR correlator.

After initial setup scans, the flux calibrator 3C48 was observed, followed by observations of the target interleaved with short observations of a standard gain calibrator, the quasar QSO B2320-035, every 10 minutes. The total resulting time on the target was $\sim 90$ minutes. Initial data editing, RFI excision, and calibration were carried out using Common Astronomy Software Applications (CASA) VLA calibration pipeline (McMullin et al. 2007). Imaging the resulting calibrated data revealed a $100 \mathrm{mJy}$ source $\sim 4$ arcmin from the position of TRAPPIST-1, limiting the rms noise to $\sim 30 \mu \mathrm{Jy}$ per beam in the region of TRAPPIST-1. This bright quasar source is evident in Figure 1. Three iterations of phase-only self-calibration followed by two subsequent iterations of phase and amplitude self-calibration resulted in rms noise in the region of TRAPPIST-1 of $2.7 \mu \mathrm{Jy}$ per beam in Stokes I and $2.6 \mu \mathrm{Jy}$ per beam in Stokes V, consistent with expectation for thermal noise.

We measure a flux density level of $3.8 \mu \mathrm{Jy}$ in the synthesized beam at the proper motion corrected position for TRAPPIST-1, consistent with the noise level. We show radio images in Stokes I and Stokes V at the location of TRAPPIST-1 in Figure 2, with no source clearly evident above the noise level. We thus report a $3 \sigma$ flux density upper limit for TRAPPIST-1 from 4 to $8 \mathrm{GHz}$ of $<8.1 \mu \mathrm{Jy}$ for our unresolved source, which corresponds to a specific luminosity of $<1.43 \times 10^{12} \mathrm{erg} \mathrm{s}^{-1} \mathrm{~Hz}^{-1}$. We performed an additional search for short duration pulses of emission by using the CASA task fixvis to shift the phase center of the data to the proper motion corrected position for TRAPPIST-1 and subtracting a model of all other sources in the field using the CASA task $u v s u b$. We then plotted the real part of the complex visibilities, averaged across all baselines, frequencies and both polarizations, representing the light curve at the location of TRAPPIST-1. No evidence for emission from TRAPPIST-1 was present in the light curve. Finally, we imaged each of the nine individual 10 minute scans on the target in Stokes I and V to search for emission on these timescales, with no evidence of any source above the $3 \sigma$ detection threshold.

\section{Stellar Emission Mechanisms}

Our observations provide the first radio flux density limits from TRAPPIST-1, allowing us to compare this result to possible expectations from different models of the magnetic activity from this star.

\subsection{Coronal Emission}

The strong X-ray emissions from TRAPPIST-1 suggest that it possesses a hot coronal atmosphere. Based on the known X-ray emission, the Güdel-Benz relation, using the linear fit to the literature data from Berger et al. (2010), would predict a radio luminosity of only $(2.2-6.0) \times 10^{10} \mathrm{erg} \mathrm{s}^{-1} \mathrm{~Hz}^{-1}$, below our measured limit of $<10^{12.2} \mathrm{erg} \mathrm{s}^{-1} \mathrm{~Hz}^{-1}$, and consistent with the solar-like paradigm of magnetic activity. Our radio limit is also an order of magnitude lower than the typical quiescent radio luminosity of the known radio emitting M7M9.5 dwarfs, which depart significantly from the Güdel-Benz relation (Williams et al. 2014; Pineda et al. 2017). TRAPPIST1 is likely very similar to other very-low-mass stars that show coronal behavior, like VB 8 or VB 10 (Hawley \& JohnsKrull 2003), which have comparable limits on their radio flux densities and exhibit X-ray emission (Berger et al. 2008). Future radio observations, however, would require an improvement of two orders of magnitude in thermal noise to confirm whether the Güdel-Benz relation continues to describe the $\mathrm{X}$-ray and radio luminosities of these very-low-mass stars. 

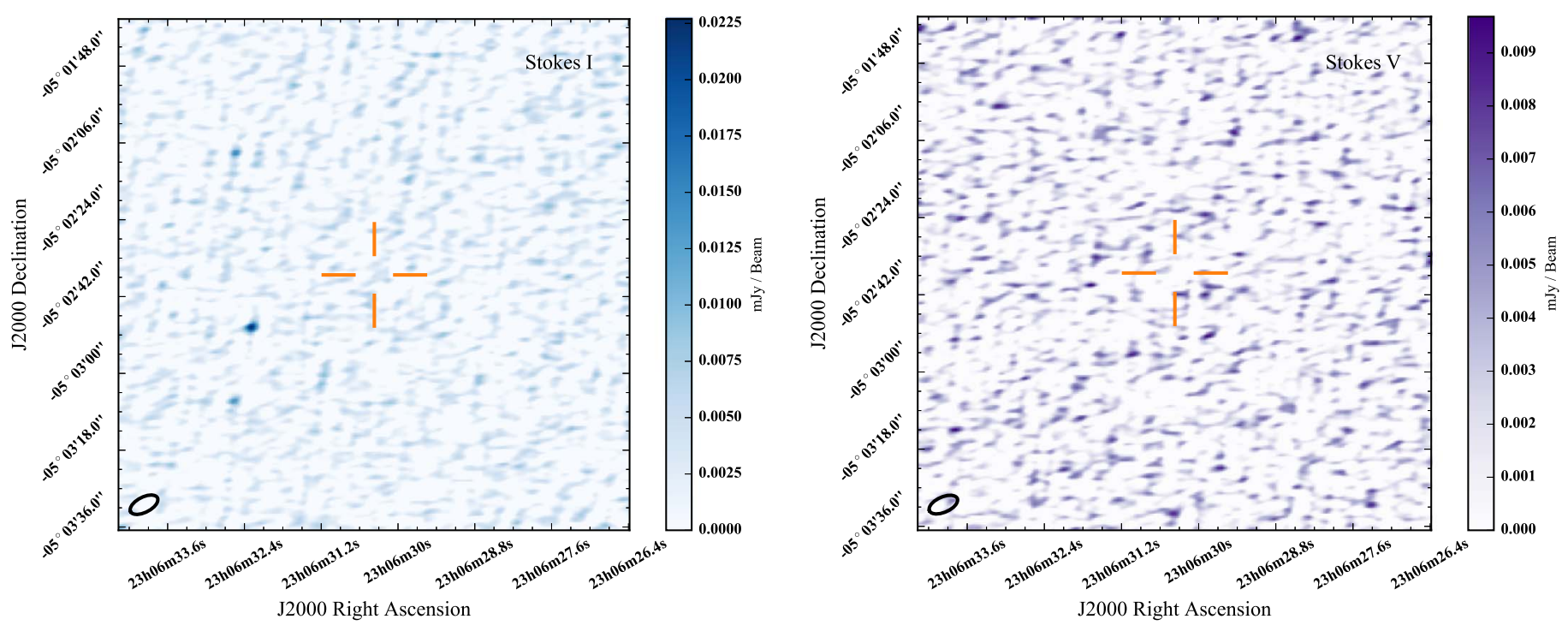

Figure 2. Left: a zoomed in view of the Stokes I radio image around the expected location of TRAPPIST-1, corresponding to the $2^{\prime}$ wide squares in Figure 1 . We do not detect a source at this position and report a $3 \sigma$ upper limit of $<8.1 \mu \mathrm{Jy}$. Right: the zoomed in view of the Stokes $\mathrm{V}$ radio image around the expected location of TRAPPIST-1, corresponding to the same $2^{\prime}$ wide squares shown in Figure 1. We do not detect any evidence for circularly polarized emission from TRAPPIST-1.

Nevertheless, these findings are in agreement with the conclusions of Stelzer et al. (2012), suggesting that among UCDs, the X-ray bright and radio loud objects are distinct populations (see also Williams et al. 2014). Building on results from McLean et al. (2012), Williams et al. (2014) proposed a magnetic field topology difference between the two populations, based on the possibility of multiple dynamo modes in this stellar regime (Morin et al. 2010). Williams et al. (2014) additionally based their conclusions on the continuity from mid-M dwarf studies showing a correlation between average surface magnetic field measurements and X-ray luminosity. Interestingly, Shulyak et al. (2017) showed that for low-mass stars with measured topologies from ZDI, either largely multipolar or dipolar, both types can display strong X-ray emission $\left(\log _{10} L_{X} / L_{\mathrm{bol}} \sim-3\right)$, with average surface fields up to $4 \mathrm{kG}$ in the multipolar case, and even larger in the dipolar case. Extending this into the UCD regime, these results would suggest that the X-ray luminosity alone cannot be used to separate the populations with different large-scale topologies. Williams et al. (2014) attributed the X-ray bright objects to the population with axisymmetric dipolar large-scale fields and the radio loud objects to those with predominantly multipolar fields. We posit that it is more likely to be the reverse, as the mechanisms theorized to produce the radio emission require strong dipolar large-scale field topologies (Pineda et al. 2017; Turnpenney et al. 2017), and in light of the Shulyak et al. (2017) results, that the X-ray emission alone cannot be used to distinguish the likely topology, the radio measurements are necessary to classify the X-ray bright and radio loud populations. Moreover, if the presence of the radio emission depends on additional factors, not just the field topology or stellar properties (see Section 5), the populations of X-ray and radio emitting objects may not be totally mutually exclusive. There is some evidence of this already in the small handful of radio UCDs with detected quiescent X-ray luminosities (Williams et al. 2014). Accordingly, these data could be explained if strong ( $\gtrsim 4 \mathrm{kG}$ surface averaged) large-scale dipolar fields are a requisite to power the radio emission, but significant X-ray emissions are possible with either topology, depending on other properties, such as $T_{\text {eff. }}$ In this scenario for
UCDs, X-ray emission measurements would not provide any information of the large-scale field topology, but the presence of radio emission would. An X-ray nondetection may suggest a weaker average surface field without constraining the topology, and a radio nondetection would similarly leave the question of topology open. ZDI measurements of the population of radio loud UCDs, confirming or refuting whether their large-scale fields are predominately axisymmetric and dipolar, would significantly help determine the relationship between these various measures of magnetic activity, and assess how the topology may differ between the populations posed by Stelzer et al. (2012).

\subsection{Stellar Wind-Planet Interaction}

Our radio flux density limits also provide new constraints on the physical parameters determining the power of its potential radio emission. Using the framework of Saur et al. (2013), Turnpenney et al. (2018) demonstrated that the planets around TRAPPIST-1 could induce significant radio emission by serving as an obstacle to a magnetized stellar wind flow from the host star. In this sub-Alfvénic interaction, the Poynting flux that dissipated across the planetary obstacle in the wind flow propagates toward the stellar surface in Alfvén waves that power the ECM instability (Turnpenney et al. 2018). We note that this scenario is not seen in the solar system, as the magnetized solar system planets interacting with the solar wind are in a super-Alfvénic regime (Zarka 2007). ${ }^{4}$ Their results indicate that TRAPPIST-1 could emit steady-state radio emission $\sim 10 \mu \mathrm{Jy}$ with possible bursts two orders of magnitude greater due to stochastic processes in the wind flow, magnetic field strength, and/or field orientation (Turnpenney et al. 2018). However, assumptions in this calculation, for example, concerning the stellar wind outflow rate or the planetary magnetic field strengths are very uncertain, and thus might be overestimating the true levels of radio emission generated. Nevertheless, with our measured flux density limit of $<8.1 \mu \mathrm{Jy}$

\footnotetext{
4 The super- and sub-Alfvénic regimes are determined by whether the wind speed exceeds the Alfvén speed of the local magnetic field.
} 
at $6 \mathrm{GHz}$, we do not observe any radio emission consistent with these estimates, and thus our observations begin to constrain the parameter space available within the TRAPPIST- 1 system to generate radio emission through this interaction between the stellar wind and the close-in planets. However, a parameter space search is beyond the scope of this work, as the combined modeling assumptions concerning the form of the magnetized stellar wind, planetary magnetic fields, and radio emission properties make constraints on the individual parameters, like the wind outflow rate or planetary dipole moment, inconsequential without additional data constraining these processes. Within the $2 \mathrm{hr}$ duration of our observations, we also do not see evidence for any possible bursts, providing a constraint on the duty cycle of the possible stochastic events that might generate bursts of radio emission.

However, an important consideration is that the ECM emission modeled by Turnpenney et al. (2018) is emitted at frequencies tied to the magnetic field strength in the vicinity of the source, presumably near the stellar surface, which has an average surface field strength of only $600 \mathrm{G}$ (Reiners \& Basri 2010), corresponding to $1.68 \mathrm{GHz}$; the lower bound of our passband at $4 \mathrm{GHz}$ corresponds to $\sim 1.43 \mathrm{kG}$ fields. This nominal field strength, $B f$, averages together both magnetic and nonmagnetic regions across the stellar surface, and thus the highly magnetized regions, such as above star spots, likely hold much stronger fields, $\sim 600 / f \mathrm{G}$. However, the filling factor is unknown in the Zeeman Broadening measurements of mid-late M dwarfs as $B$ and $f$ are not separable (Reiners \& Basri 2007), making the maximum surface field strengths uncertain. Although the average field strength corresponds to frequencies lower than our observing band, ECM emission may still be generated up to the maximum field strengths on the stellar surface, possibly encompassed by our $4-8 \mathrm{GHz}$ observations. If there were ECM source regions tied to field lines with these higher field strengths, we may have expected to see this kind of emission based on the estimates from Turnpenney et al. (2018). The lack of a detection could be explained by a paucity of magnetic field regions exceeding strengths $\sim 1.5 \mathrm{kG}$, our observing frequencies being potentially too high to probe the weaker magnetic fields of TRAPPIST-1. A ZDI map of the this star's magnetic field topology would help determine whether this was indeed the case. Alternatively, even if these regions were prevalent, there is no guarantee that these also corresponded to ECM source regions. A deep search at lower frequencies than our observations is warranted to rule out or potentially detect ECM generated from this star-planet interaction.

Additionally, due to the beaming of the ECM emission into less than $4 \pi$ sr (Zarka et al. 2004; Treumann 2006), our observations may not have been optimally oriented in space or time to intercept the emission. Although the beaming geometry of hollow and wide ECM source cones near the rotational axis favors observability when viewed near an inclination of $90^{\circ}$ (Pineda et al. 2017), which is likely the case if the planetary system angular momentum and magnetic axes are aligned with the stellar rotation axis, the rotational and orbital phases need to be well sampled to rule out ECM emission entirely-the $2 \mathrm{hr}$ extent of our observations is much smaller than the several day rotation and orbital periods of TRAPPIST-1 and its planetary system.

\subsection{Auroral Emission}

Although we did not see any highly polarized pulsations, the limited rotational phase coverage $(\sim 3 \%)$ in our observations cannot rule them out; nevertheless, our measured upper limit on the flux density of quiescent radio emission does provide information on the likelihood of possible GHz ECM emission from TRAPPIST-1, given the statistical association of quiescent and pulsed emission among UCDs (Pineda et al. 2017). Although the underlying cause is uncertain, Pineda et al. (2017) demonstrated a correlation between the observed quiescent radio luminosity at $\mathrm{GHz}$ frequencies, likely of synchrotron origin (Ravi et al. 2011; Williams et al. 2015b), and $\mathrm{H} \alpha$ luminosity among known periodically pulsing radio UCDs. If TRAPPIST-1 behaved like those auroral objects, we would expect a radio luminosity of $\sim 10^{13.8} \mathrm{erg} \mathrm{s}^{-1} \mathrm{~Hz}^{-1}$, but instead our measured limit of $<10^{12.2} \mathrm{erg} \mathrm{s}^{-1} \mathrm{~Hz}^{-1}$ is over a magnitude smaller, well below that relationship even accounting for the scatter of $\sim 0.24 \mathrm{dex}$, at fixed $\mathrm{H} \alpha$ luminosity. Even if TRAPPIST-1 exhibited radio emission at levels below our detection threshold, the star would inhabit a region of $L_{\mathrm{H} \alpha}-L_{\nu \text {, rad }}$ space with other very-low-mass stars that have their $\mathrm{H} \alpha$ luminosities dominated by chromospheric emission, instead of being associated with the presence of auroral currents (Pineda et al. 2017). Our results thus confirm that $\mathrm{H} \alpha$ emission is not a viable proxy for quiescent radio emission in the TRAPPIST-1 system, it is not auroral and instead is likely dominated by chromospheric emission, and that TRAPPIST-1 is not likely to generate radio emission in the same manner as the known auroral radio UCDs.

\section{Role of Planets in Producing UCD Radio Emission}

Our aim in conducting these observations was to provide a deep limit for quiescent radio emission from the TRAPPIST-1 system in the context of both stellar radio emission and auroral processes in the UCD regime. We further aimed to test whether the presence of a planetary system is a crucial ingredient to the production of strong $^{5}$ radio emission from UCDs, either through a direct star-planet interaction that produces ECM emission or by the detection of quiescent radio emission associated with the presence of magnetospheric currents possibly due to equatorial radiation belts. While our null detection in this instance points toward the coronal paradigm for TRAPPIST-1 and leaves the question of possible starplanet interactions open, if there is a crucial role for close-in planetary companions, our results point to the necessity of multiple conditions that must be met to produce strong radio emitters (Güdel-Benz deviants, see Section 4.1) in the population of very-low-mass stars and brown dwarfs. Such close-in satellites may or may not be necessary, but their presence is certainly not sufficient to drive the electrodynamic engines of radio UCDs.

\subsection{Magnetospheric Mass-loading}

All of the proposed hypotheses for the electrodynamic engine driving UCD radio emission (see Secton 1), except reconnection with the ISM, require a significant source of plasma internal to the system. Similarly, the radiation belts that

\footnotetext{
5 By "strong," we are referring to radio emissions exceeding the Güdel-Benz relation predictions by several orders of magnitude, which for UCDs could be $L_{\nu} \sim 10^{13.5} \mathrm{erg} \mathrm{s}^{-1} \mathrm{~Hz}^{-1}$, although there is a broad range, see Section 4.1 and Williams et al. (2014).
} 
might explain the quiescent emission of these systems requires the magnetosphere to be loaded with plasma. Using the Jovian system as an example, a volcanic planet, like Io, can be this source. However, our radio observations show no evidence for these magnetospheric plasma structures around TRAPPIST-1. This could be a consequence of either of two distinct possibilities: either the planetary satellites are not providing sufficient plasma to the magnetosphere, or the magnetosphere does not sustain large-scale loops with which to contain the plasma. Even though the equilibrium tidal heating of the TRAPPIST-1 planets from N-body simulations suggests internal heat fluxes comparable or potentially greater than that of Io (Luger et al. 2017), the volcanism on these planets may not be contributing to the mass-loading of the magnetosphere. One reason may be that the larger masses of the TRAPPIST-1 planets (Wang et al. 2017; Grimm et al. 2018) relative to the Galilean moons prevent significant amounts of volcanic material from escaping the planetary atmospheres. Even if a strong stellar wind, as suggested by Garraffo et al. (2017), can erode the planetary atmospheres, the material would likely be entrained with the wind along the open field lines, instead of populating a steady-state plasma torus like that observed in the Jovian magnetosphere (e.g., Bagenal et al. 1997). Any material that does populate the magnetospheric environment must be trapped in closed large-scale magnetic loops in order to create an equatorial radiation belt of synchrotron emission. Without a significant large-scale component to the stellar magnetic field, the ability of such stars to generate very strong quiescent radio emission similar to that observed from the known radio UCDs may be limited, irrespective of any mass-loading.

\subsection{Jupiter-Io Analogs in UCD Systems}

Our radio observations of TRAPPIST- 1 also provide a test of the possibility of magnetic flux tube interactions analogous to the Jupiter-Io system powering radio emission from UCDs. Our nondetection suggests these processes, if powering the known radio UCDs, are not taking place in the TRAPPIST-1 system, or are too weak to detect. One reason for this is likely associated with the slow TRAPPIST-1 rotation rate, relative to known radio UCDs, as well as possibly the role of large-scale magnetic field topologies. This is evident in models of the subAlfvénic interaction driving the Jupiter-Io current system responsible for Io-related Jovian decametric ECM radio emission (Saur et al. 2013). The relevant equation for the total surface integrated Poynting flux ${ }^{6}$ generated by the differential motion of a planetary body embedded in a large magnetosphere in the limit of small Alfvén mach numbers (small velocities relative to the Alfvén speed) from Saur et al. (2013) is

$$
S_{\text {total }}=\frac{1}{2} \bar{\alpha}^{2} R_{o}^{2} v^{2} B \sin ^{2} \theta \sqrt{4 \pi \rho}
$$

where $\bar{\alpha}$ is the dimensionless interaction strength, ${ }^{7} R_{o}$ is the effective radius of the planet obstacle defined by the planetary magnetosphere or ionosphere, $B$ is the magnetic field strength from the star in the vicinity of the planet in Gaussian units, $v$ is the relative speed of the planet through the stellar magnetosphere,

\footnotetext{
6 For convenience and to reflect on its origins, we refer to the output of Equation (1) as the "Poynting flux"; however, the quantity has units of luminosity.

$7 \bar{\alpha} \sim 0.5$ for the Galilean satellites (Saur et al. 2013) but could be near unity for the TRAPPIST-1 planets (Turnpenney et al. 2018).
}

$\theta$ is the angle between the magnetic field and the relative velocity vector, and $\rho$ is the mass density of the plasma environment. While the presence of a planet provides the necessary obstacle, and potentially supplies a sufficiently dense plasma environment, a strong stellar generated magnetic field and rapidly rotating magnetosphere are also necessary.

To illustrate this, we consider, for the innermost planet of the TRAPPIST-1 system, the Poynting flux generated according to Equation (1), if the star hosted a large-scale dipolar field consistent with its measured average surface field (see Table 1). We take $\bar{\alpha} \rightarrow 1, \theta=90^{\circ}$, and plasma densities similar to values around Io, with a number density of $\sim 2000 \mathrm{~cm}^{-3}$ and mean molecular weight of 22 amu (Saur et al. 2013). The radius of the obstacle is taken as at minimum, the planetary radius $1.127 R_{\oplus}$ of TRAPPIST-1b (Delrez et al. 2018). The velocity is the relative velocity between the orbital motion and the rotating magnetosphere at the location of the planet, $v \sim 45 \mathrm{~km} \mathrm{~s}^{-1}$. The field strength at the location of the planet is $\sim 0.05 \mathrm{G}$, assuming the planet lies along the magnetic equator. Plugging in these values gives a Poynting flux of $\sim 2.5 \times 10^{13} \mathrm{~W}$. Assuming a conversion efficiency of $1 \%$ from Poynting flux to radio power, a beam solid angle of $1.6 \mathrm{sr}$ (Zarka et al. 2004; Turnpenney et al. 2018), and using a $4 \mathrm{GHz}$ bandwidth consistent with EMC observations of UCDs (Hallinan et al. 2015), this would correspond to a radio flux density of $\sim 0.03 \mu \mathrm{Jy}$, which is well below current radio observatory capabilities. This flux density drops further if the 1.4 day period is used since that is very near the orbital period of TRAPPIST-1b. If instead we consider the same planet in a 1 day orbit around a UCD like TRAPPIST- 1 but with a $2 \mathrm{hr}$ rotation period, like that observed from radio UCDs (Pineda et al. 2017), and $5 \mathrm{kG}$ average surface field strength with a dipolar large-scale field topology consistent with ZDI measurements (Shulyak et al. 2017), the corresponding flux density of ECM emission would be $\sim 300 \mu \mathrm{Jy} \quad\left(L_{\nu, \text { rad }}=\right.$ $\left.10^{12.6} \mathrm{erg} \mathrm{s}^{-1} \mathrm{~Hz}^{-1}\right)$, readily detectable. Although this estimate is smaller than the strength of some of the observed highly circularly polarized radio bursts from UCDs (see Table 1 of Pineda et al. 2017), it is subject to many unknown quantities, including the radio emission efficiency of the ECM instability $(\sim 0.01)$, and the beaming solid angle $(\sim 1.6 \mathrm{sr})$, in addition to other system properties like the plasma environment. For example, while a beaming solid angle of $\sim 1.6 \mathrm{sr}$ is commonly used as a basis for estimating ECM radio fluxes from UCDs (Nichols et al. 2012; Turnpenney et al. 2017), it could be as low as $~ 0.16$ sr (Queinnec \& Zarka 2001; Zarka et al. 2004), which would increase the predicted flux by a factor of 10 . Our estimates based on Equation (1) are linear in the magnetic field strength, so a factor of 10 weaker field $(500 \mathrm{G})$, with the same rapid rotation could still produce detectable emission $(\sim 30 \mu \mathrm{Jy})$; however, if the rotation is slightly slower or the plasma environment is less dense, the prospects for currently detectable emission become marginal. Additionally, if the field is mostly multipolar, the field strength at the planet location would drop off more quickly with distance, further limiting the strength of these potential emissions. Nevertheless, even if TRAPPIST-1 generated ECM emission like the Jupiter-Io system, assuming an optimistic large-scale field topology, it would have been too weak to detect with our current observations. Several kG surface magnetic field strengths in dipolar topologies, and fast rotation rates $(\sim 2 \mathrm{hr})$ are necessary to generate currently detectable $\mathrm{GHz}$ frequency $\mathrm{ECM}$ radio 
emission through a star-planet flux tube interaction, like that of Jupiter and Io. Conversely, if the TRAPPIST-1 planetary system was hosted by one of the rapidly rotating UCDs with strong ( $\gtrsim 4 \mathrm{kG}$ surface averaged) large-scale dipolar magnetic fields, then the system would generate ECM emission consistent with the emission levels observed in many of the known radio UCDs. Given the estimates of planet occurrence rates in short period orbits around very-low-mass stars $(\sim 30 \%$; He et al. 2017), and the rapid rotation rates of most UCDs with likely strong dipolar fields, the overall radio UCD detection rate may be plausibly determined by the presence of these three conditions.

\section{Conclusions}

Our observations of the TRAPPIST-1 system centered at $6 \mathrm{GHz}$ yielded no detectable radio emission to a limit of $<8.1 \mu$ Jy. In Section 4.1, we demonstrated that this limit was consistent with the Güdel-Benz relation, applicable to coronally active low-mass stars, and motivated future ZDI observations of radio loud UCDs to discern the role that magnetic field topology plays in dictating the activity indicators in this stellar population. In Section 4.2, we compared our radio measurements to the possible strength of ECM emission driven by an impinging stellar wind, as calculated by Turnpenney et al. (2018), concluding that we did not see any such ECM emission from the TRAPPIST-1 system, possibly due to low rotational/orbital phase coverage, with further observations, including at lower frequencies, required to rule out the possibility of wind driven ECM emission. In Section 4.3, we also compared this limit to possible quiescent emission levels based on correlations among the population of known radio UCDs (Pineda et al. 2017), illustrating that TRAPPIST-1 does not likely exhibit detectable radio emission that behaves in the same manner as the emission from the known periodically pulsing radio UCDs.

Although we cannot use these new data on TRAPPIST-1 to discern the role, if any, that planetary systems have in generating bright radio emissions in the population of very-low-mass stars and brown dwarfs, it is evident that the presence of a planetary system by itself does not guarantee strong radio emission at $\mathrm{GHz}$ frequencies. If the observed ECM radio emissions of UCDs are generated through a magnetic interaction analogous to the JupiterIo system (see Section 5.2), additional criterion beyond the presence of a close-in planet must also be met, namely rapid rotation and strong (several $\mathrm{kG}$ surface averaged) magnetic field strengths - the TRAPPIST-1 system does not satisfy these latter two conditions. Additionally, even if planets are present around UCDs, their capacity to provide a source of magnetospheric plasma is still an open question depending on the tidal heating and volcanism of those planets. Similarly, the retention of such a magnetospheric plasma likely depends on the magnetic field topology and the presence of large-scale magnetic loops. These loops may then serve as the site for the persistent quiescent synchrotron emission.

In light of the ZDI observations of fully convective low-mass stars showing two regimes of dynamos and their respective large-scale field topologies, either predominantly dipolar or multipolar, with the strongest fields associated with the dipolar topology and fastest rotators (Shulyak et al. 2017), the connection between observed ECM and quiescent radio emission could be explained as a coherent consequence of this strong large-scale dipolar field when a sufficient plasma source is available. The $\mathrm{kG}$ magnetic field strengths help power the UCD auroral electrodynamic engine (Turnpenney et al. 2017) and the closed large-scale field houses the magnetospheric plasma that generates the quiescent emission. These assorted criteria may then collectively contribute to the low detection statistics for UCD radio emission (e.g., Lynch et al. 2016). Future surveys looking for UCD radio emission at $\mathrm{GHz}$ frequencies are more likely to succeed targeting the fastest rotating objects, while the slower rotators or objects with significant X-ray emission may be better targeted at lower frequencies, hundreds of MHz. TRAPPIST-1, as a slow rotator, with strong coronal X-ray and weak radio emissions, and likely possessing a multipolar large-scale field, reflects the population of very-low-mass stars with coronal solar-like activity instead of the subpopulation exhibiting auroral magnetic processes. We suggest that the X-ray emission of a given object alone is not a sufficient indication of the likely field topology, but in conjunction with radio emission measurements can provide an indication of that topology to the extent that it is responsible for the dichotomy of observed X-ray and radio properties among UCDs. This is potentially powerful as ZDI measurements of these objects are difficult with current instrumentation due to the faint intrinsic luminosities and rapid rotation of UCDs. Nevertheless, these assessments remain circumstantial; new measurements of the magnetic field topology of UCDs are necessary to definitively establish this connection between the magnetic emissions and the field topology.

It remains to be seen whether the strong ECM and quiescent radio emissions of the few radio UCDs is related to the presence of planets, or are predominantly driven without them (Turnpenney et al. 2017). While the magnetic field strength and rotation rate of TRAPPIST-1 are consistent with the nondetection of radio emission within a Jupiter-Io flux tube paradigm, it is notable that the same planetary configuration orbiting a rapidly rotating dwarf with a large-scale dipolar field can account for the observed radio luminosities of radio emitting UCDs. The prevalence of multiplanet systems in tight orbits orbiting UCDs, as well as the fraction of rapid rotators with strong magnetic fields, may prove to be consistent with the detection rate of radio pulsed emission from these systems. However, such evidence would be circumstantial with direct confirmation requiring detection of radio pulses from a TRAPPIST-1 like system. More deep radio searches in soon to be discovered planetary systems around very-low-mass stars and brown dwarfs, as well as potential planet detections among the growing population of radio detected UCDs will help elucidate the answer.

J.S.P. would like to thank Kevin France, Zach BertaThompson, and Jackie Villadsen for helpful comments in the preparation of this manuscript. G.H. acknowledges the generous support of NSF Career award AST-1654815. The authors would also like to thank the anonymous referee for the thoughtful consideration of this manuscript and for providing comments that strengthened this article.

The National Radio Astronomy Observatory is a facility of the National Science Foundation operated under cooperative agreement by Associated Universities, Inc.

Funding for SDSS-III has been provided by the Alfred P. Sloan Foundation, the Participating Institutions, the National Science Foundation, and the U.S. Department of Energy Office of Science. The SDSS-III website is http://www.sdss3.org/. 
SDSS-III is managed by the Astrophysical Research Consortium for the Participating Institutions of the SDSS-III Collaboration including the University of Arizona, the Brazilian Participation Group, Brookhaven National Laboratory, Carnegie Mellon University, University of Florida, the French Participation Group, the German Participation Group, Harvard University, the Instituto de Astrofisica de Canarias, the Michigan State/Notre Dame/JINA Participation Group, Johns Hopkins University, Lawrence Berkeley National Laboratory, Max Planck Institute for Astrophysics, Max Planck Institute for Extraterrestrial Physics, New Mexico State University, New York University, Ohio State University, Pennsylvania State University, University of Portsmouth, Princeton University, the Spanish Participation Group, University of Tokyo, University of Utah, Vanderbilt University, University of Virginia, University of Washington, and Yale University.

Software: Aladin (Bonnarel et al. 2000), CASA (McMullin et al. 2007).

\section{ORCID iDs}

J. Sebastian Pineda (iD https://orcid.org/0000-0002-4489-0135

\section{References}

Ahn, C. P., Alexandroff, R., Allende Prieto, C., et al. 2012, ApJS, 203, 21 Alibert, Y., \& Benz, W. 2017, A\&A, 598, L5

Antonova, A., Hallinan, G., Doyle, J. G., et al. 2013, A\&A, 549, A131

Bagenal, F., Crary, F. J., Stewart, A. I. F., et al. 1997, GeoRL, 24, 2119

Barstow, J. K., \& Irwin, P. G. J. 2016, MNRAS, 461, L92

Berger, E., Basri, G., Gizis, J. E., et al. 2008, ApJ, 676, 1307

Berger, E., Basri, G., Fleming, T. A., et al. 2010, ApJ, 709, 332

Bonnarel, F., Fernique, P., Bienaymé, O., et al. 2000, A\&As, 143, 33

Bourrier, V., de Wit, J., Bolmont, E., et al. 2017, AJ, 154, 121

Burgasser, A. J., \& Mamajek, E. E. 2017, ApJ, 845, 110

Burgasser, A. J., Logsdon, S. E., Gagné, J., et al. 2015, ApJS, 220, 18

de Wit, J., Wakeford, H. R., Lewis, N. K., et al. 2018, NatAs, 2, 214

Delrez, L., Gillon, M., Triaud, A. H. M. J., et al. 2018, MNRAS, 475, 3577

Garraffo, C., Drake, J. J., Cohen, O., Alvarado-Gómez, J. D., \& Moschou, S. P. 2017, ApJL, 843, L33

Gillon, M., Jehin, E., Lederer, S. M., et al. 2016, Natur, 533, 221

Gillon, M., Triaud, A. H. M. J., Demory, B.-O., et al. 2017, Natur, 542, 456

Grimm, S. L., Demory, B.-O., Gillon, M., et al. 2018, A\&A, 613, A68

Guedel, M., \& Benz, A. O. 1993, ApJL, 405, L63
Hallinan, G., Antonova, A., Doyle, J. G., et al. 2006, ApJ, 653, 690 Hallinan, G., Littlefair, S. P., Cotter, G., et al. 2015, Natur, 523, 568 Hawley, S. L., \& Johns-Krull, C. M. 2003, ApJ, 588, L109

He, M. Y., Triaud, A. H. M. J., \& Gillon, M. 2017, MNRAS, 464, 2687

Luger, R., Sestovic, M., Kruse, E., et al. 2017, NatAs, 1, 0129

Lynch, C., Murphy, T., Ravi, V., et al. 2016, MNRAS, 457, 1224

McLean, M., Berger, E., \& Reiners, A. 2012, ApJ, 746, 23

McMullin, J. P., Waters, B., Schiebel, D., Young, W., \& Golap, K. 2007, in ASP Conf. Ser. 376, Astronomical Data Analysis Software and Systems XVI, ed. R. A. Shaw, F. Hill, \& D. J. Bell (San Francisco, CA: ASP), 127

Morin, J., Donati, J.-F., Petit, P., et al. 2010, MNRAS, 407, 2269

Morley, C. V., Kreidberg, L., Rustamkulov, Z., Robinson, T., \& Fortney, J. J. 2017, ApJ, 850, 121

Morris, B. M., Agol, E., Davenport, J. R. A., \& Hawley, S. L. 2018, ApJ, 857,39

Nichols, J. D., Burleigh, M. R., Casewell, S. L., et al. 2012, ApJ, 760, 59

Perley, R. A., Chandler, C. J., Butler, B. J., \& Wrobel, J. M. 2011, ApJL, 739, L1

Pineda, J. S., Hallinan, G., \& Kao, M. M. 2017, ApJ, 846, 75

Pineda, J. S., Hallinan, G., Kirkpatrick, J. D., et al. 2016, ApJ, 826, 73

Queinnec, J., \& Zarka, P. 2001, P\&SS, 49, 365

Ravi, V., Hallinan, G., Hobbs, G., \& Champion, D. J. 2011, ApJL, 735, L2

Reiners, A., \& Basri, G. 2007, ApJ, 656, 1121

Reiners, A., \& Basri, G. 2010, ApJ, 710, 924

Reiners, A., Zechmeister, M., Caballero, J. A., et al. 2018, A\&A, 612, A49

Roettenbacher, R. M., \& Kane, S. R. 2017, ApJ, 851, 77

Saur, J., Grambusch, T., Duling, S., Neubauer, F. M., \& Simon, S. 2013, A\&A, 552, A119

Schrijver, C. J. 2009, ApJL, 699, L148

Shulyak, D., Reiners, A., Engeln, A., et al. 2017, NatAs, 1, 0184

Stelzer, B., Alcalá, J., Biazzo, K., et al. 2012, A\&A, 537, A94

Treumann, R. A. 2006, A\&Ar, 13, 229

Turnpenney, S., Nichols, J. D., Wynn, G. A., \& Burleigh, M. R. 2018, ApJ, 854,72

Turnpenney, S., Nichols, J. D., Wynn, G. A., \& Casewell, S. L. 2017, MNRAS, 470, 4274

Van Grootel, V., Fernandes, C. S., Gillon, M., et al. 2018, ApJ, 853, 30

Vida, K., Kővári, Z., Pál, A., Oláh, K., \& Kriskovics, L. 2017, ApJ, 841,124

Wang, S., Wu, D.-H., Barclay, T., \& Laughlin, G. P. 2017, arXiv:1704.04290

Wheatley, P. J., Louden, T., Bourrier, V., Ehrenreich, D., \& Gillon, M. 2017, MNRAS, 465, L74

Williams, P. K. G., Berger, E., Irwin, J., Berta-Thompson, Z. K., \& Charbonneau, D. 2015a, ApJ, 799, 192

Williams, P. K. G., Casewell, S. L., Stark, C. R., et al. 2015b, ApJ, 815,64

Williams, P. K. G., Cook, B. A., \& Berger, E. 2014, ApJ, 785, 9

Zarka, P. 2007, P\&SS, 55, 598

Zarka, P., Cecconi, B., \& Kurth, W. S. 2004, JGRA, 109, A09S15 